DRAFT VERSion August 22, 2018

Preprint typeset using $\mathrm{LT}_{\mathrm{E}} \mathrm{X}$ style emulateapj v. 6/22/04

\title{
TIMING OF MILLISECOND PULSARS IN NGC 6752 - II. PROPER MOTIONS OF THE PULSARS IN THE CLUSTER OUTSKIRTS
}

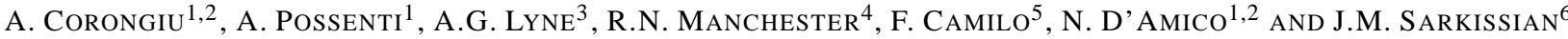 \\ Draft version August 22, 2018
}

\begin{abstract}
Exploiting a five-year span of data, we present improved timing solutions for the five millisecond pulsars known in the globular cluster NGC 6752. They include proper motion determinations for the two outermost pulsars in the cluster, PSR J1910-5959A and PSR J1910-5959C. The values of the proper motions are in agreement with each other within current uncertainties, but do not match (at $4 \sigma$ and $2 \sigma$ level respectively) with the value of the proper motion of the entire globular cluster derived in the optical band. Implications of these results for the cluster membership of the two pulsars are investigated. Prospects for the detection of the Shapiro delay in the binary system J1910-5959A are also discussed.
\end{abstract}

Subject headings: pulsars: individual (PSR J1910-5959A; PSR J1910-5959B; J1910-5959C; J1910-5959D; J1910-5959E) - globular clusters: individual (NGC 6752)

\section{INTRODUCTION}

The globular cluster (GC) NGC 6752 is known to host five millisecond pulsars (MSPs) (D'Amico et al. 2001; D’Amico et al. 2002, hereafter Paper I). PSR J1910-5959B and PSR J1910-5959E (hereafter PSR B and E respectively) reside in the central region of the GC and show large negative $\dot{P}$ values, which are interpreted as an effect of the GC gravitational potential well (Paper I). This in turn implies a large mass-to-light ratio in the central region of NGC 6752. Ferraro et al. (2003b) recalculated the center of gravity and studied the luminosity profile of this cluster: combining their HST data with the $\dot{P}$ value of PSR B and PSR E, they put a firm lower limit on the central mass-to-light ratio of $M / L_{V} \gtrsim 5.5 \mathrm{M}_{\odot} / \mathrm{L}_{\odot}$. Also PSR J1910-5959D (PSR D) is located close to the GC center. Its $\dot{P}$ value is positive and of the same order of magnitude of PSR B and E, suggesting that also for PSR D the $\dot{P}$ value is dominated by the gravitational potential well (Paper I). PSR J1910-5959C ${ }^{7}$ (PSR C) is located at a projected distance $\theta_{\perp}=2$ '.6 from the GC center (Paper I), which is much larger than the cluster's core radius $r_{c}=5$ !. $2 \pm 2$ !" 4 (Ferraro et al. 2003b). The binary pulsar J1910-5959A (PSR A) is located at an even larger distance from the GC center $\left(\theta_{\perp}=6 ! 4\right.$, Paper I), the largest offset known for a GC pulsar.

The positions of PSR A and PSR C are unexpected since mass segregation should have driven the two neutron stars close to the GC center in a time scale $(\lesssim 1 \mathrm{Gyr})$ much shorter than the time since their formation $(\sim 10 \mathrm{Gyr})$. In particular, Colpi et al. (2002, 2003) explored various scenarios to

\footnotetext{
1 INAF - Osservatorio Astronomico di Cagliari, Loc. Poggio dei Pini, Strada 54, 09012 Capoterra (CA), Italy

${ }^{2}$ Università degli Studi di Cagliari, Dip. di Fisica, S.S. Monserrato-Sestu km 0,700, 09042 Monserrato (CA), Italy

${ }^{3}$ University of Manchester, Jodrell Bank Observatory, Jodrell Bank, Macclesfield, Cheshire, SK11 9DL, UK

4 Australia Telescope National Facility, Commonwealth Scientific and Industrial Research Organization, P.O. Box 76, Epping, NSW 1710, Australia

5 Columbia Astrophysics Laboratory, Columbia University, 550 West, 120th Street, New York, NY 10027

${ }^{6}$ Australia Telescope National Facility, CSIRO, Parkes Observatory, P.O. Box 276, Parkes, New South Wales 2870, Australia

${ }^{7}$ Note that, to conform with currently accepted practice, all pulsars associated with the cluster have been given a J2000 name with the same rounded coordinates, corresponding approximately to the cluster centre.
}

explain the unusual position of PSR A, invoking a dynamical encounter in the inner region of the GC. The most probable picture is that PSR A was originally in the GC central regions and it has been expelled to the outskirts by the interaction with either a single massive black hole $(\mathrm{BH})$ or a binary $[\mathrm{BH}+\mathrm{BH}]$ of unequal mass. Timing results in Paper I indicated a low-mass white dwarf as the most probable companion for PSR A. This has been confirmed by Bassa et al. (2003) and Ferraro et al. (2003a), who identified with Hubble Space Telescope observations the companion of PSR A with a helium white dwarf star whose mass is $M_{c o} \simeq 0.17-0.20 \mathrm{M}_{\odot}$ and whose photometric properties are compatible with its belonging to NGC 6752.

The issue of the association of PSR A to NGC 6752 has been recently revisited using spectroscopic observations of the optical companion to the pulsar, performed with the ESOVLT. Cocozza et al. (2006) found full agreement (at $1 \sigma$ ) between the radial velocity of the center of mass of the binary $\gamma=-28.1 \pm 4.9 \mathrm{~km} \mathrm{~s}^{-1}$ and the overall cluster radial velocity $v_{6752}=-27.9 \pm 0.8 \mathrm{~km} \mathrm{~s}^{-1}$, obtained by Harris (1996) (cata$\log$ revision 2003) averaging various determinations. This is a strong indication in favour of the association of the pulsar with NGC 6752. However, using the same data set, Bassa et al. (2006) compared the systemic velocity of the binary with that of nearby stars which certainly belong to the cluster and concluded that they are only marginally consistent at $2 \sigma$ level.

In this paper we present timing results based on more than five years of regular observations. In particular, with a much longer available data span we have been able to measure proper motions of PSR A and PSR C. The new timing solutions as well as the pulse profiles for all the millisecond pulsars are presented in $\S 2, \S 3$ reports on the proper motion determinations and implications for the cluster membership of the two pulsars are discussed in $\S 4$.

\section{OBSERVATIONS AND IMPROVED TIMING PARAMETERS}

Regular pulsar timing observations of NGC 6752 have been carried out since September 2000 with the Parkes $64 \mathrm{~m}$ radio telescope at a central frequency of $1390 \mathrm{MHz}$, using the central beam of the multibeam receiver or the $\mathrm{H}-\mathrm{OH}$ receiver. The hardware system is the same as that used in the discovery observations (D'Amico et al. 2001). The effects of interstellar dispersion are minimised by using a filterbank having 
$512 \times 0.5 \mathrm{MHz}$ frequency channels for each polarization. After detection, the signals from individual channels are added in polarization pairs, integrated, 1 bit-digitized every $125 \mu \mathrm{s}$ ( $80 \mu$ s in recent observations), and recorded to magnetic tape for off-line analysis. Pulse times of arrival (TOAs) are determined by fitting a template profile to the observed mean pulse profiles and analysed using the program TEMPO 8 and the DE405 solar system ephemeris.

Table 1 summarizes the best fit values and uncertainties (chosen to be twice the nominal TEMPO errors) for the parameters entering our timing solutions, whose residuals are displayed in Figure 1 The same figure presents a high signalto-noise profile obtained for each of the pulsars by folding the best available data according to the reported ephemerides.

The new positional and rotational parameters at the reference epoch are all compatibile with those reported in Paper I (assuming $3 \sigma$ uncertainties for the values quoted in Paper I). However, the MJD range of the available TOAs is now $\sim 3.5$ times longer than for Paper I and hence the accuracy of the solutions has improved correspondingly. Orbital parameters for PSR A, obtained using the ELL1 model of TEMPO, have also been measured with a higher precision than in Paper I. Figure 2 shows that no trend is evident in the timing residuals plotted with respect to the orbital phase for the timing solution given in Table 1 An additional constraint on the orbit of PSR A has resulted from the recent optical observations of the pulsar companion. Spectroscopy (Cocozza et al. 2006; Bassa et al. 2006) has provided us with a measurement of the mass ratio in the binary, whereas multi-color photometry has set the possible range for the mass of the secondary star (Ferraro et al. 2003a; Bassa et al. 2006). Combining these results gives a limit on the orbital inclination $i \gtrsim 70^{\circ}$ (Cocozza et al. 2006; Bassa et al. 2006).

The size of the expected Shapiro delay is nominally larger than the rms residual of the timing solution (see Table 1 for any $i \gtrsim 70^{\circ}$, but, except for inclination angles near $90^{\circ}$, a large part of the Shapiro delay is absorbed in the Roehmer delay (Lange et al.2001). In fact, no clear trend is visible in the timing residuals even after binning the TOAs in orbital phase (see Figure 2), indicating that the magnitude of the unabsorbed component of Shapiro delay is below the present uncertainty in the TOAs. Therefore it is not surprising that fitting the available TOAs with TEMPO has not led to any significant determination of the Shapiro parameter $s$. Inspection of Figure 2 also shows that the present uncertainties on the TOAs allow us only to exclude very extreme orbital inclinations $i \gtrsim 89^{\circ}$. Simulations show that a factor $\sim 2-3$ improvement in timing precision is needed in order to obtain a useful constraint on $s$. This will require an additional $\sim 10$ years of observation with the present instrumentation and collection rate of TOAs.

The still unassessed effects of Shapiro delay may also affect the new determination of the binary eccentricity, for which in Paper I only an upper limit was available. Neglecting Shapiro delay, the measured value is $e=3.4(12) \times 10^{-6}$ (here and everywhere in this paper the errors are quoted at twice the nominal rms values given by TEMPO). However, for $70 \lesssim i \lesssim 89^{\circ}$ and $0.17 \mathrm{M}_{\odot} \lesssim M_{c o} \lesssim 0.20 \mathrm{M}_{\odot}$ an unmodeled Shapiro delay can introduce an apparent eccentricity in the range $1-3 \times 10^{-6}$. The determination of $e$ must still be considered provisional and $e=4.6 \times 10^{-6}$ is a reliable upper limit.

\footnotetext{
${ }^{8}$ see http://www.atnf.csiro.au/research/pulsar/timing/tempo
}

The small eccentricity of PSR A's binary system is typical of fully recycled binary millisecond pulsars and is consistent with the effects of random encounters with other cluster stars (Rasio \& Heggie 1995). The upper limit on $e$ is also compatible with the offset position of PSR A resulting from an interaction which occurred $\sim 1$ Gyr ago between the already recycled binary system including PSR A and a WD companion [PSR A+WD] with a binary black hole of a few tens of solar masses (Colpi et al. 2003, see $\S 1$ ). We note that the value of $e$ also fits with the hypothesis (Bassa et al. 2003; Colpi et al. 2003) that a dynamical encounter with a single $\mathrm{BH}$, whose mass is higher than a few hundred $\mathrm{M}_{\odot}$, may have simultaneously ejected the progenitor of [PSR A+WD] and triggered the recycling process in the binary, which in turn circularized the system and removed any information on its post-encounter eccentricity. However, the value of $e$ does not agree with an ejection event involving the already formed [PSR A + WD] binary and a single BH. In this case, Colpi et al. (2003) showed that the post-encounter eccentricity of [PSR A+WD] would be significantly larger, up to values of $10^{-4}-10^{-2}$ and only slightly affected by subsequent random encounters with normal stars of the cluster (Rasio \& Heggie 1995).

The mean flux densities at $1400 \mathrm{MHz}\left(S_{1400}\right)$ in Table 1 are average values, derived from the system sensitivity, the observed signal-to-noise ratio, the shape of the pulse profile, the displacement of the pulsars with respect to the center of the telescope beam and assuming flux density values corresponding to half the detection limit for the non-detections due to the strong interstellar scintillation effects on the pulsars in NGC 6752 (see $\S 3$ ). The uncertainties on the values of $S_{1400}$ may reach $\sim 30 \%$ for the faintest sources. For a distance $d=4.45 \pm 0.15 \mathrm{kpc}$ (Gratton et al. 2003) the inferred radio luminosities at $1400 \mathrm{MHz}$ of the two millisecond pulsars in the cluster's outskirts are $L_{1400}=S_{1400} d^{2} \sim 4-5 \mathrm{mJy} \mathrm{kpc}^{2}$, a value in the middle of the distribution of the luminosities of the millisecond pulsars in 47 Tucanae (Camilo et al. 2000).

\section{PROPER MOTION DETERMINATIONS}

The main improvement in our timing solutions is that proper motion determinations for the two outermost pulsars in NGC 6752 are now available. In Table 1 proper motion components in right ascension and declination are reported as well as the corresponding proper motion amplitude and position angle (PA, measured counterclockwise from north toward east). Proper motion uncertainties depend on the length of the data span and on the number, the degree of uniformity and the errors of the TOAs along the data span. The different precisions in our measurements are mainly due to the different number of high quality TOAs available for each pulsar, as shown in Table 1 Measurement of good TOAs for the faintest pulsars is possible only when interstellar scintillation enhances their signal: this is the reason that in the timing analysis of PSRs B, D, and E we used a significantly smaller number of TOAs than for PSR A. The flux density of PSR C is similar to that of PSR A and the effects of interstellar scintillation are also comparable. The difference in rms residual between the timing solutions for these two pulsars is primarily due to the different pulse widths, being $\sim 7$ times larger (at $50 \%$ of the peak) for PSR C than for PSR A.

Figure 3 presents a geometrical representation of the expected motion in the plane of the sky (during next $10^{4} \mathrm{yrs}$ ) of PSR A, PSR C, and of the center of NGC 6752, as derived from their measured proper motions. The proper motion for the center of the globular cluster has been obtained 
by Dinescu et al. (1999), by comparing two optical observations taken 25 years apart. The values for the components are $\mu_{\alpha} \cos \delta=-0.7 \pm 0.8 \mathrm{mas} \mathrm{yr}^{-1}$ and $\mu_{\delta}=-2.9 \pm 0.9 \mathrm{mas} \mathrm{yr}^{-1}$. Their derivation required a transformation of the coordinate system at the epoch of the first observation to the coordinate system at the epoch of the second observation and the use of distant field galaxies as reference. The inset in Figure 3 shows a comparison between the proper motion vectors of PSR A and PSR C (with their uncertainties), and the optical proper motion vector of the cluster. The proper motions of PSR A and PSR C are compatible with each other, but they are not in agreement with the optical proper motion of NGC 6752, at $4 \sigma$ and $2 \sigma$ confidence levels respectively.

\section{DISCUSSION}

Since the escape velocity from a globular cluster is usually significantly lower than the typical transverse velocity of these stellar systems, it is expected that the proper motion of a cluster pulsar will largely reflect the overall motion of the cluster. For NGC 6752, the escape velocity from the central region is $\sim 30 \mathrm{~km} \mathrm{~s}^{-1}$ (Colpi et al. 2003) and the space velocity is $\sim 62 \mathrm{~km} \mathrm{~s}^{-1}$ with respect to the Solar System barycenter, based on the proper motion measurement by Dinescu et al. (1999) and the distance derived from the distance modulus (Gratton et al. 2003). Observations over a much longer data span may reveal the peculiar (orbital) motion of a pulsar in the cluster's gravitational potential well.

Is it possible that the discrepancy between the proper motions of PSR A and PSR C and the optical proper motion of NGC 6752 (§3) could be an indication that the two pulsars are not associated to the globular cluster?

In Paper I it was estimated that the probability ${ }^{9}$ for PSR A to be a Galactic field millisecond pulsar superposed by chance to NGC 6752 (at a distance of 6.4 off its center) is of order $10^{-5}$. The compatibility of the measured proper motions of PSR A and PSR C reinforces the unlikeliness for these two MSP to be Galactic field objects by chance superimposed to the globular cluster.

Assuming that both PSRs $\mathrm{A}$ and $\mathrm{C}$ are members of NGC 6752, the discrepancy between pulsar and GC proper motions, measured in the radio and optical band respectively, may result from the different methods used for determining the proper motions in the two spectral bands. In fact, similar discrepancies have already been noted for the pulsars in 47 Tucanae (Freire et al. 2001, 2003), in M4 (Thorsett et al. 1999) and, more recently, in M15 (Jacoby et al. 2006).

However, the discrepancies in these clusters may not easily be ascribed to a common systematic effect affecting all the optical measurements. The optical proper motion for 47 Tucanae was directly measured based on Hipparcos observations. The proper motion determination for M4 (Cudworth \& Hanson 1993) was based on the determination of its motion relative to a set of reference field stars whose proper motion relative to the Sun has been in turn obtained by combining their position in the Galaxy (through their parallax) to a dynamic model for the nearby regions of the Galaxy where these reference stars reside. Finally, the proper motion for NGC 6752 (Dinescu et al. 1997) resulted from the comparison of two photographic plates taken

\footnotetext{
${ }^{9}$ It is worth nothing that this probability does not account for the similar values of the dispersion measure of PSR A and PSR C. Given the uncertainty in the Galactic electron layer, it is difficult to quantify the probability for this coincidence (Bassa et al. 2006). However, it certainly further decreases the total probability for a chance superposition.
}

25 years apart, using distant field galaxies as reference objects. In the case of M15, four different optical determinations have been performed (Geffert et al.|1993; Scholz et al. 1996; Odenkirchen et al. 1997; Cudworth \& Hanson 1993), three of which are incompatible with the pulsar proper motions. These three non-matching measures were respectively based on the comparison between photographic plates at different epochs (Geffert et al. 1993; Scholz et al. 1996) and the use of reference stars from Hipparcos observations (Odenkirchen et al. 1997). Only the measurement by Cudworth \& Hanson (1993) is in agreement with the apparent motions of the three pulsars investigated. However it is worth noting that Cudworth \& Hanson (1993) measured the optical proper motion for M15 applying the same method as was used for M4, which in that case led to a discrepant proper motion.

The discrepancy may be alternatively ascribed to very fast peculiar motions of PSR A and PSR C inside the cluster gravitational potential well. Assuming that both the pulsar proper motions and the optical proper motion of the cluster are correct, the relative 2-D velocity vectors of the pulsars with respect to the cluster center are roughly directed towards the cluster inner regions, as is indicated in Figure 3 This would mean that [PSR A+WD] cannot be now in the phase of ejection from the cluster and that it is not at its farthest distance from the GC center along its orbit inside the cluster gravitational well. For $d=4.45 \pm 0.15 \mathrm{kpc}$ (Gratton et al. 2003) the relative transverse speed of PSR A would be $V_{\text {rel,A }}=$ $51 \pm 16 \mathrm{~km} \mathrm{~s}^{-1}$. NGC 6752 can provide a gravitational pull strong enough to retain PSR A at its actual location with a peculiar velocity $V_{\text {rel,A }}$ only if the mass enclosed within the pulsar projected position is $M_{\text {encl }} \geq 1.18 \times 10^{6} \mathrm{M}_{\odot}$. This is in contrast to the total mass value for the cluster obtained with HST observation (Sabbi et al. 2004), which is lower by an order of magnitude. Using again the distance modulus in Gratton et al. (2003), the apparent magnitude given by Harris (1996) and the colour excess $E_{\mathrm{B}-\mathrm{V}}=0.04$ in Ferraro et al. (1999), the resulting overall mass-to-light ratio would be $M_{\text {encl }} / L \geq 8.4 \mathrm{M}_{\odot} / \mathrm{L}_{\odot}$, which is unreasonably high for a GC, unless we admit an initial mass function much flatter than usually estimated (so producing a very large number of underluminous stellar remnants) or the presence in the cluster of a significant amount of dark matter. At a more conservative confidence level $(4 \sigma)$ for the relative velocity $V_{\text {rel,A }}$, the mass to light ratio would result $M_{\text {encl }} / L \geq 2.5 \mathrm{M}_{\odot} / \mathrm{L}_{\odot}$, again implying a dynamic mass much higher than the mass derived from optical observations.

A further test of the cluster membership of PSR A and PSR C will be possible in future. It will involve the comparison of the proper motions of PSR A and PSR C with those of the three pulsars close to the cluster core, whose association with NGC 6752 is unambigously proved by the very strong gravitational pull affecting the values of their spin period derivative. This task will take some years: our simulations show that, with the present accuracy and collection rate of the TOAs and if the three innermost pulsars display the same proper motion as PSR A, a $3 \sigma$ determination will require a total data span of about $8-10$ years.

AC, AP and NDA acknowledge the financial support to this reasearch provided by the Ministero dell'Istruzione, dell'Università $e$ della Ricerca (MIUR) under the national program PRIN05 2005024090_002. The Parkes radio telescope is part of the Aus- 


\section{REFERENCES}

Bassa, C. G., van Kerkwijk, M. H., Koester, D., \& Verbunt, F. 2006, ArXiv Astrophysics e-prints

Bassa, C. G., Verbunt, F., van Kerkwijk, M. H., \& Homer, L. 2003, A\&A 409, L31

Camilo, F., Lorimer, D. R., Freire, P., Lyne, A. G., \& Manchester, R. N. 2000, ApJ, 535, 975

Cocozza, G., Ferraro, F. R., Possenti, A., \& D’Amico, N. 2006, ApJ, 641, L129

Colpi, M., Mapelli, M., \& Possenti, A. 2003, ApJ, 599, 1260

Colpi, M., Possenti, A., \& Gualandris, A. 2002, ApJ, 570, L85

Cudworth, K. M. \& Hanson, R. B. 1993, AJ, 105, 168

D’Amico, N., Lyne, A. G., Manchester, R. N., Possenti, A., \& Camilo, F. 2001, ApJ, 548, L171

D’Amico, N., Possenti, A., Fici, L., Manchester, R. N., Lyne, A. G., Camilo, F., \& Sarkissian, J. 2002, ApJ, 570, L89

Damour, T. \& Taylor, J. H. 1991, ApJ, 366, 501

Dinescu, D. I., Girard, T. M., \& van Altena, W. F. 1999, AJ, 117, 1792

Dinescu, D. I., Girard, T. M., van Altena, W. F., Mendez, R. A., \& Lopez, C. E. 1997, AJ, 114, 1014

Ferraro, F. R., Messineo, M., Fusi Pecci, F., de Palo, M. A., Straniero, O., Chieffi, A., \& Limongi, M. 1999, AJ, 118, 1738

Ferraro, F. R., Possenti, A., Sabbi, E., \& D’Amico, N. 2003a, ApJ, 596, L211

Ferraro, F. R., Possenti, A., Sabbi, E., Lagani, P., Rood, R. T., D’Amico, N. \& Origlia, L. 2003b, ApJ, 595, 179

Freire, P. C., Camilo, F., Kramer, M., Lorimer, D. R., Lyne, A. G., Manchester, R. N., \& D'Amico, N. 2003, MNRAS, 340, 1359

Freire, P. C., Camilo, F., Lorimer, D. R., Lyne, A. G., Manchester, R. N., \& D'Amico, N. 2001, MNRAS, 326, 901
Geffert, M., Colin, J., Le Campion, J.-F., \& Odenkirchen, M. 1993, AJ, 106, 168

Gratton, R. G., Bragaglia, A., Carretta, E., Clementini, G., Desidera, S., Grundahl, F., \& Lucatello, S. 2003, A\&A, 408, 529

Harris, W. E. 1996, AJ, 112, 1487, updated version at http://www.physics.mcmaster.ca/resources/globular.html

Jacoby, B. A., Cameron, P. B., Jenet, F. A., Anderson, S. B., Murty, R. N., \& Kulkarni, S. R. 2006, ArXiv Astrophysics e-prints

Lange, C., Camilo, F., Wex, N., Kramer, M., Backer, D., Lyne, A., \& Doroshenko, O. 2001, MNRAS, 326, 274

Odenkirchen, M., Brosche, P., Geffert, M., \& Tucholke, H. J. 1997, New Astronomy, 2, 477

Phinney, E. S. 1992, Philos. Trans. Roy. Soc. London A, 341, 39

Rasio, F. R. \& Heggie, D. C. 1995, ApJ, 445, L133

Sabbi, E., Ferraro, F. R., Sills, A., \& Rood, R. T. 2004, ApJ, 617, 1296

Scholz, R.-D., Odenkirchen, M., Hirte, S., Irwin, M. J., Borngen, F., \& Ziener, R. 1996, MNRAS, 278, 251

Shklovskii, I. S. 1970, Sov. Astron., 13, 562

Thorsett, S. E., Arzoumanian, Z., Camilo, F., \& Lyne, A. G. 1999, ApJ, 523, 763

Trager, S., Djorgovski, S., \& King, I. 1993, in Structure and dynamics of globular clusters, ed. S. Djorgovski \& G. Meylan, Vol. 50 (ASp Conference series), 347 


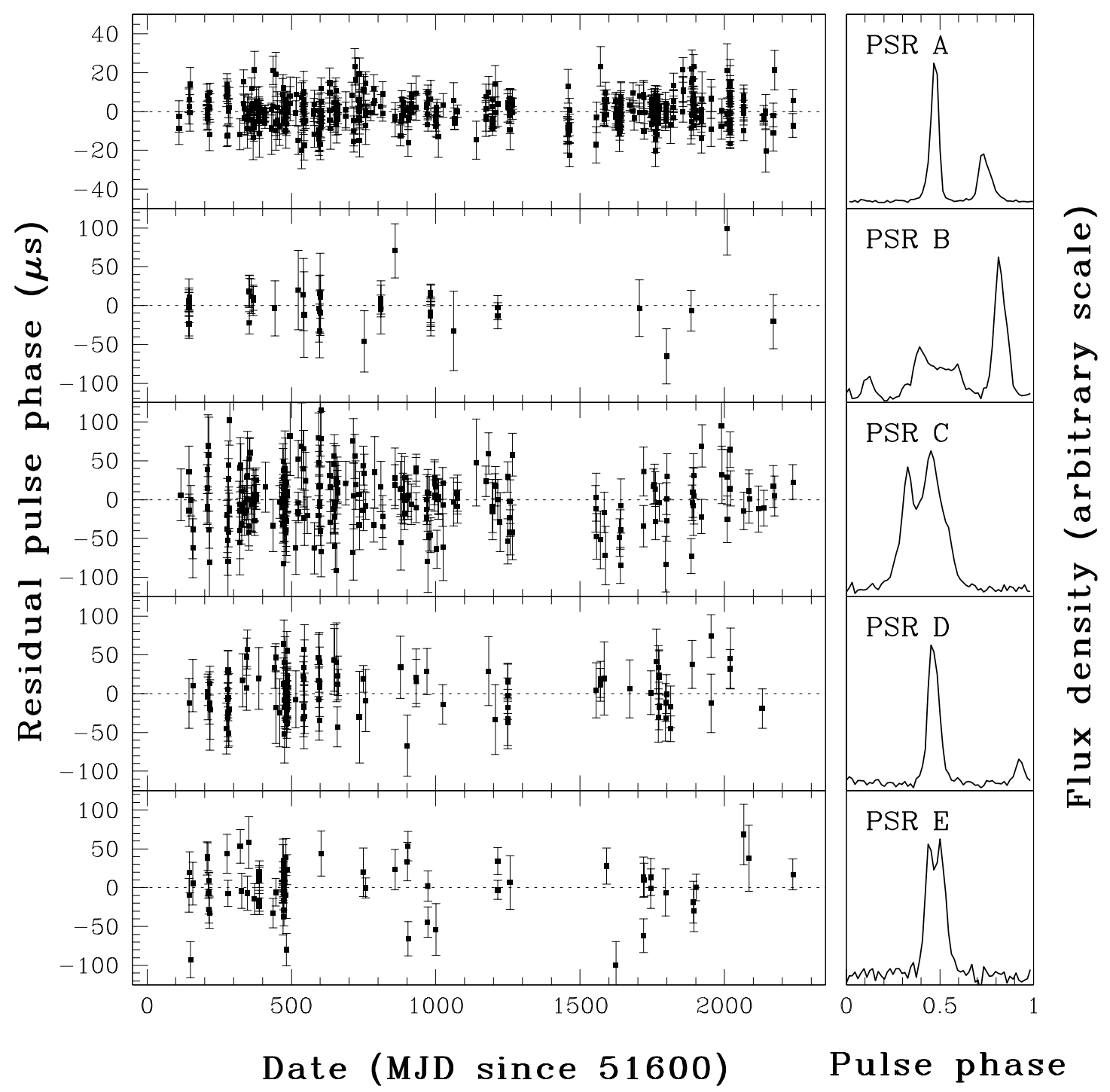

FIG. 1.- Fit residuals (left panels) and pulse profiles (right panels) for the five pulsars known in NGC 6752. Mean pulse profiles shown in the right panels are the sum of the observed profiles with the highest signal-to-noise ratio. The adopted binning (64 bins) matches the time resolution of the profiles. 


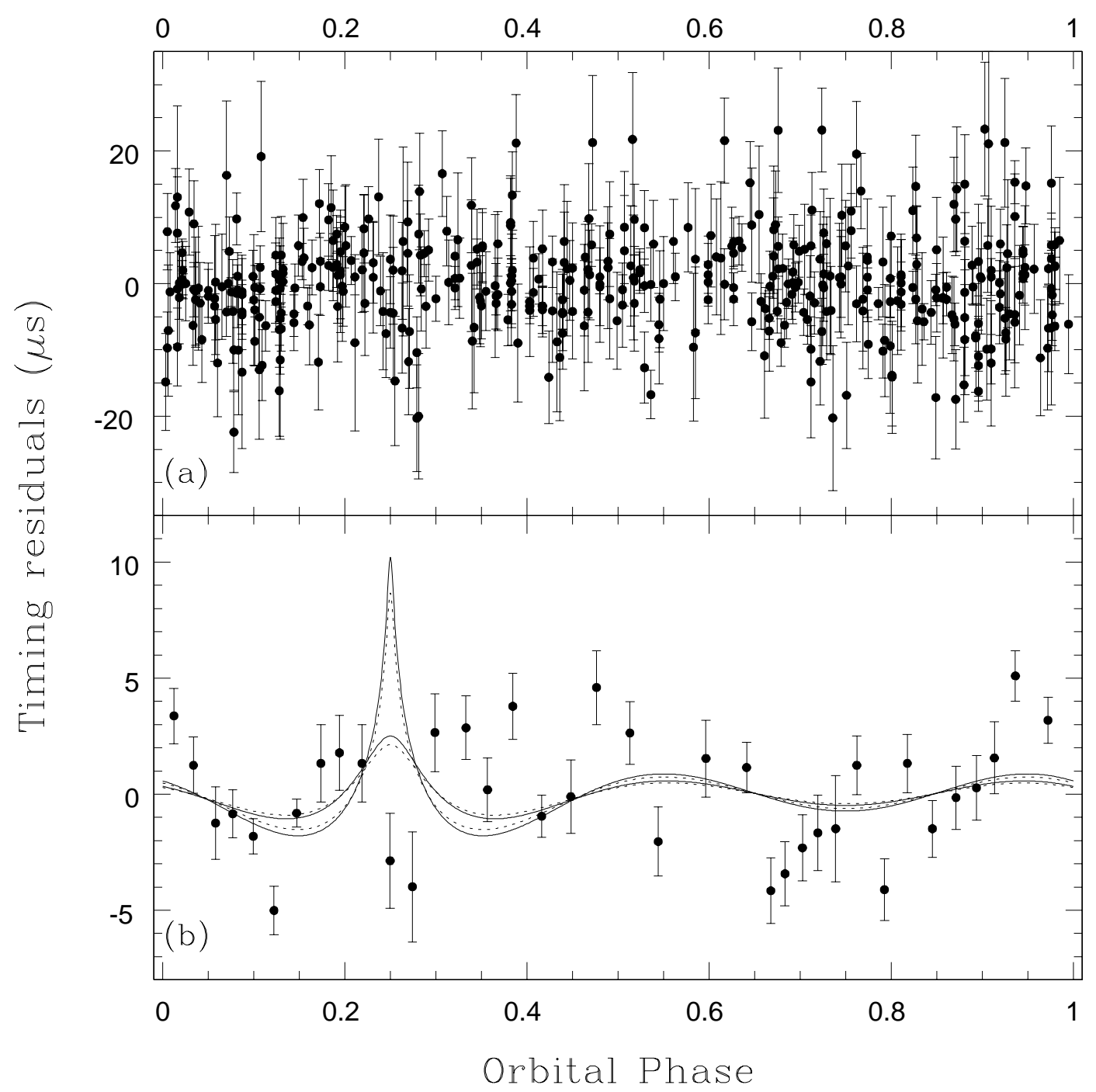

FIG. 2.- (a) Fit residuals versus orbital phase for PSR A obtained from the timing solution of Table 1 (b) Timing residuals binned in 42 orbital bins. The central values and the plotted uncertainties result from a weighted average (and error propagation) performed on all the available TOAs in each orbital bin. The lines represent the expected trends of the timing residuals when the Shapiro delay is not included in the timing model. The two upper curves are for an orbital inclination $i=89^{\circ}$, whereas the two lower curves are for $i=80^{\circ}$. The mass of the companion star is taken as $0.20 \mathrm{M}_{\odot}$ (solid line) and $0.17 \mathrm{M}_{\odot}($ dotted line) in both sets of curves. 


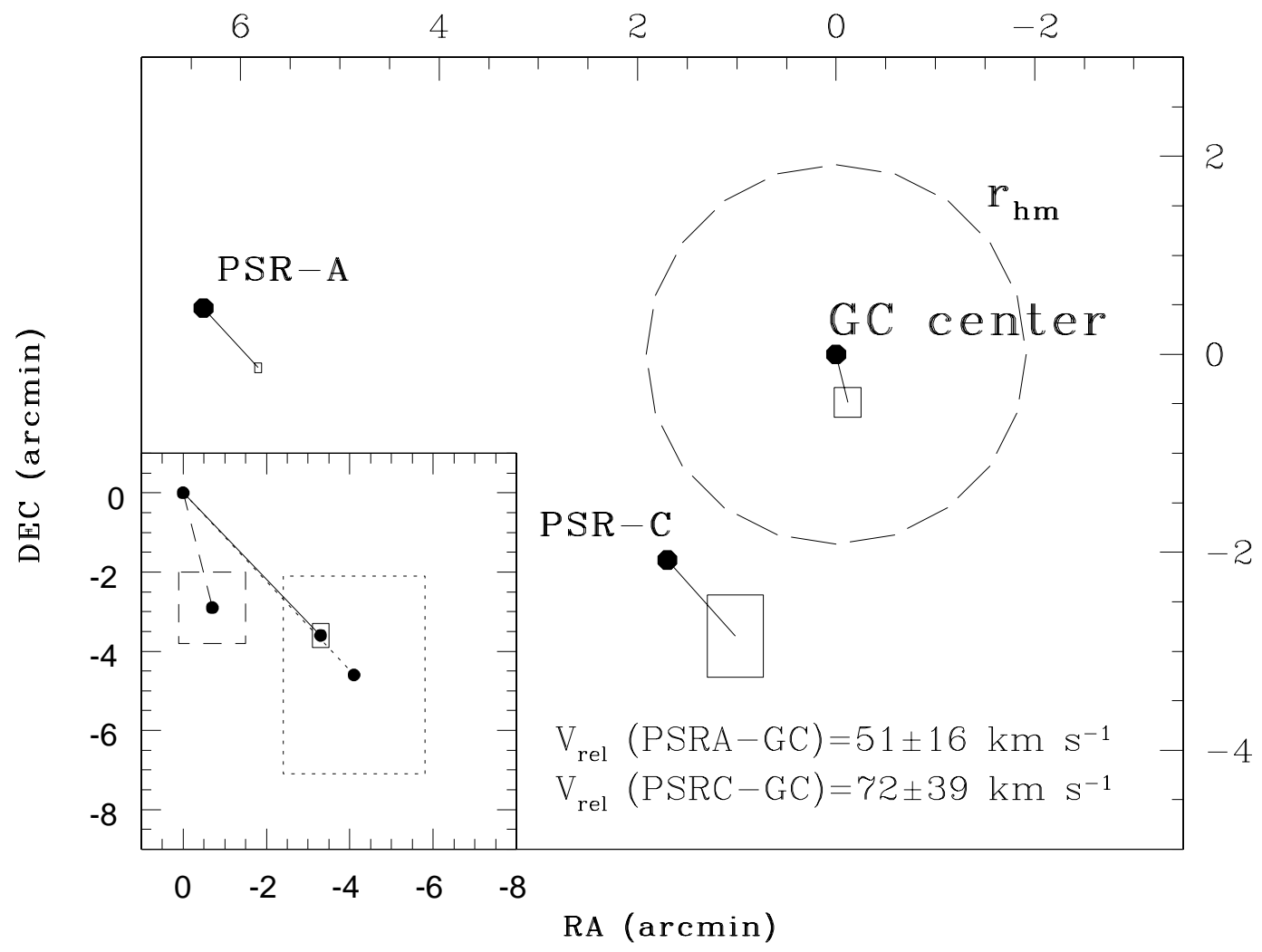

FIG. 3.- Main panel - Positions and expected changes (assuming uniform motion) after $10^{4}$ years for PSR A, PSR C and the center of NGC 6752 relative to the present position of the cluster center. The uncertainties in the expected final positions are described by boxes whose size is given by the propagation of the uncertainties on the proper motions in right ascension and declination ( $2 \sigma$ confidence level). Proper motion uncertainties for the pulsars are from Table 1 while the uncertainties for the optical proper motion of the cluster are from Dinescu et al. (1999). The dashed circle represents the portion of the cluster enclosed within the half-mass radius $r_{\mathrm{hm}}=1^{\prime}$.9 (Trager et al. 1993). Lower-left panel - Comparison of the motions of the two outermost pulsars (PSR A: solid line; PSR C: dotted line)and the globular cluster (dashed line) relative to their present position. 
TABLE 1

MEASURED AND DERIVED PARAMETERS FOR THE PULSARS IN NGC 6752.

\begin{tabular}{|c|c|c|c|c|c|}
\hline Parameter & PSR A & PSR B & PSR C & PSR D & PSR E \\
\hline R.A. (J2000) & $19: 11: 42.75562(8)$ & $19: 10: 52.0556(5)$ & 19:11:05.5552(4) & $19: 10: 52.4163(5)$ & $19: 10: 52.1572(6)$ \\
\hline Decl. (J2000) & $-59: 58: 26.904(1)$ & $-59: 59: 00.861(6)$ & $-60: 00: 59.700(4)$ & $-59: 59: 05.479(5)$ & $-59: 59: 02.087(7)$ \\
\hline$\mu_{\alpha} \cos \delta\left(\right.$ mas $\left.\mathrm{yr}^{-1}\right)$ & $-3.3(2)$ & - & $-4.1(17)$ & - & - \\
\hline$\mu_{\delta}\left(\operatorname{mas} \mathrm{yr}^{-1}\right)$ & $-3.6(3)$ & - & $-4.6(25)$ & - & - \\
\hline$\mu\left(\operatorname{mas} \mathrm{yr}^{-1}\right)$ & $4.8(3)$ & - & $6.2(22)$ & - & - \\
\hline $\mathrm{PA}^{a}(\mathrm{deg})$ & $222(3)$ & - & $221(20)$ & - & - \\
\hline$P(\mathrm{~ms})$ & $3.2661865707908(1)$ & $8.357798500844(2)$ & $5.2773269323093(15)$ & $9.035285247765(4)$ & $4.571765939750(2)$ \\
\hline$\dot{P}^{b}\left(\mathrm{~s} \mathrm{~s}^{-1}\right)$ & $2.947(2) \times 10^{-21}$ & $-7.9041(5) \times 10^{-19}$ & $2.16(2) \times 10^{-21}$ & $9.6431(6) \times 10^{-19}$ & $-4.3435(3) \times 10^{-19}$ \\
\hline Epoch (MJD) & 51920.0000 & 52000.0000 & 51910.0000 & 51910.0000 & 51910.0000 \\
\hline $\mathrm{DM}\left(\mathrm{pc} \mathrm{cm^{-3 }}\right)$ & $33.705(3)$ & $33.33(6)$ & $33.29(5)$ & $33.28(2)$ & $33.31(3)$ \\
\hline$P_{o r b}$ (days) & $0.8371134769(1)$ & - & - & - & - \\
\hline$a \sin i(1-\mathrm{s})$ & $1.2060461(8)$ & - & - & - & - \\
\hline$T_{a s c}(\mathrm{MJD})$ & $51919.20647998(16)$ & - & - & - & - \\
\hline$e \sin \omega$ & $3.3(12) \times 10^{-6}$ & - & - & - & - \\
\hline$e \cos \omega$ & $0.9(13) \times 10^{-6}$ & - & - & - & - \\
\hline$f\left(M_{\mathrm{c}}\right)\left(\mathrm{M}_{\odot}\right)$ & $0.002687854(6)$ & - & - & - & - \\
\hline $\mathrm{M}_{c, \min }{ }^{c}\left(\mathrm{M}_{\odot}\right)$ & 0.19 & - & - & - & - \\
\hline MJD Range & $51710-53836$ & $51745-53769$ & $51710-53836$ & $51745-53731$ & $51744-53836$ \\
\hline Number of TOAs & 450 & 44 & 246 & 124 & 70 \\
\hline r.m.s. residuals $(\mu \mathrm{s})$ & 5.0 & 18 & 29 & 24 & 25 \\
\hline Offset $^{d}(\operatorname{arcmin})$ & 6.37 & 0.06 & 2.56 & 0.05 & 0.05 \\
\hline$W_{10}^{e} @ 10 \%(\mathrm{~ms})$ & 0.6 & 1.3 & 2.8 & 1.1 & 1.1 \\
\hline$W_{50}^{f} @ 50 \%(\mathrm{~ms})$ & 0.4 & 0.6 & 1.3 & 0.7 & 0.6 \\
\hline$S_{1400}(\mathrm{mJy})$ & 0.21 & 0.05 & 0.24 & 0.05 & 0.07 \\
\hline
\end{tabular}

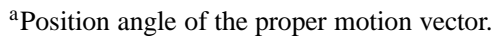

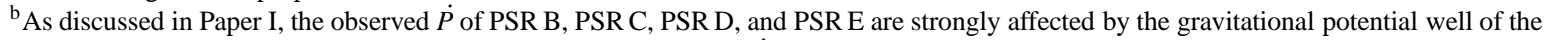
globular cluster. Useful constraints on the instrinsic spin-down rate $\dot{P}_{i}$ can hence be inferred only for PSR A. Correcting the observed value of $\dot{P}$ for $(i)$ the Galactic differential rotation and the vertical acceleration in the Galactic potential (see e.g. Damour \& Tavlor 1991), for (ii) the centrifugal acceleration of the pulsar (Shklovskii 1970), and for (iii) the contribution of the cluster potential well (estimated according to the recipe of Phinnev (1992) and using the luminosity density profile of NGC 6752 published by Ferraro et al. (2003b)) gives $\dot{P}_{i} \lesssim 6 \times 10^{-21}$ $\mathrm{s} \mathrm{s}^{-1}$. We have also adopted $M / L_{V}=5.5$ (Ferraro et al. 2003b) in order to obtain the firmest upper limit on the intrinsic spin-down rate of PSR A. This translates to a lower limit to the pulsar spin-down age of $0.5\left(P / \dot{P}_{i}\right) \sim 8.6$ Gyr and upper limits to the surface dipole magnetic field of $3.2 \times 10^{19} \sqrt{P \dot{P}_{i}} \sim 1.4 \times 10^{8} \mathrm{G}$ and to the spin-down luminosity of $4 \pi^{2} I \dot{P}_{i} / P^{3}=6.9 \times 10^{33} \mathrm{erg} \mathrm{s}^{-1}$ ( $I$ being the moment of inertia of the neutron star, set equal to $10^{45} \mathrm{~g} \mathrm{~cm}^{2}$ ).

${ }^{c}$ The minimum mass is calculated assuming a pulsar mass of $1.35 \mathrm{M}_{\odot}$ and an inclination for the orbital plane with respect to the line of sight of $90^{\circ}$.

${ }^{\mathrm{d}}$ The offset of the pulsars is calculated with respect to the position of the cluster's center of gravity reported by Ferraro et al. (2003b).

${ }^{\mathrm{e}}$ Pulse width at $10 \%$ of the height of the main peak.

${ }^{\mathrm{f}}$ Pulse width at $50 \%$ of the height of the main peak. 